\title{
Uso de membrana amniótica no tratamento de complicações
} pós-trabeculectomia

\author{
Amniotic membrane in the treatment of post-trabeculectomy complications
}

$\begin{array}{llll}\text { José } & \text { Álvaro } & \text { Pereira } \text { Gomes }^{1} \\ \text { Luiz } & \text { Henrique } & \text { Schurig } \quad \text { Fernandes }^{2} \\ \text { Ciro } & \text { Massayuki } \quad \text { Komagome } & \\ \text { Ana } & \text { Luísa } & \text { Höfling-Lima }{ }^{3} \\ \text { João } & \text { Antônio } & \text { Prata } \quad \mathrm{Jr}^{4}\end{array}$

Este trabalho foi realizado nos Setores de Glaucoma e Doenças Externas/Córnea do Depto. de Oftalmologia da Universidade Federal de São Paulo (UNIFESP).

${ }^{1}$ Doutor em oftalmologia e colaborador do Setor de Doenças Externas e Córnea do Departamento de Oftalmologia da Universidade Federal de São Paulo (UNIFESP).

${ }^{2}$ Ex-residente de $3^{\text {a }}$ ano do Depto. de Oftalmologia da UNIFESP.

${ }^{3}$ Professora livre-docente em oftalmologia e colaboradora do Setor de Doenças Externas e Córnea do Departamentode Oftalmologia da UNIFESP.

${ }^{4}$ Doutor em oftalmologia e chefe do Setor de Glaucoma do Departamento de Oftalmologia da UNIFESP.

Endereço para correspondência: Sabará, 566 apto. 212 - São Paulo (SP) CEP 01239-010. E-mail: japgomes @uol.com.br

INTRODUÇÃO

A utilização de substâncias antimetabólicas associadas a trabeculectomia melhorou o controle da pressão intra-ocular nos casos de glaucoma crônico simples e secundário refratários aos tratamentos convencionais. Entretanto, o uso dessas substâncias tem aumentado o número de complicações pós-operatórias ${ }^{(1)}$. Dentre essas complicações, destaca-se a formação de bolhas grandes, finas e hiperfiltrantes, que induzem hipotonia e irritação ocular; e o vazamento de humor aquoso com teste de Seidel +, que ocorre geralmente no pós-operatório precoce e induz hipotonia, câmara anterior rasa e dificuldade na formação da bolha filtrante ${ }^{(1)}$. Complicações graves podem ocorrer tardiamente nesses casos, como endoftalmite infecciosa e maculopatia hipotônica ${ }^{(1-2)}$.

O tratamento da bolha hiperfiltrante e do vazamento de humor aquoso é semelhante e inclui curativo oclusivo, lente de contato terapêutica com ou sem uso de cola de cianoacrilato, aplicação de ácido tricloroacético, cauterização focal, fotocoagulação com ou sem corantes para aumentar a absorção do laser, crioterapia, uso de supressores de produção do humor aquoso, enxerto lamelar com esclera de cadáver, injeção de sangue autólogo na bolha filtrante, revisão cirúrgica e diferentes técnicas de sutura ${ }^{(1-7)}$. Os resultados são muitas vezes desanimadores, pois a manipulação dessas 
bolhas filtrantes avasculares, com paredes finas e friáveis, acaba por esgarçar a conjuntiva que freqüentemente necessita ser ressecada e substituída por um novo tecido ${ }^{(7)}$. Diferentes técnicas de retalhos e transplante autólogo de conjuntiva são normalmente realizados nesses $\operatorname{casos}^{(7)}$. Entretanto, duas considerações importantes devem ser levadas em conta: 1) Deve-se procurar evitar a manipulação de locais que potencialmente possam ser utilizados para futuras cirurgias filtrantes. 2) Muitos desses pacientes já foram submetidos a várias cirurgias envolvendo a conjuntiva e não apresentam conjuntivas viáveis para ser utilizada.

Mais recentemente, o transplante da membrana amniótica foi reintroduzido na reconstrução da superfície ocular nos casos de doenças cicatriciais da córnea e conjuntiva; na cicatrização dos defeitos epiteliais persistentes; como enxerto após a remoção cirúrgica de pterígio e tumores; nas reconstruções palpebrais; e no tratamento de bolhas filtrantes com vazamento $^{(8-15)}$.

O objetivo deste trabalho é avaliar o uso de membrana amniótica para os casos de complicações pós-trabeculectomia com mitomicina.

MÉTODOS

\section{Pacientes}

Foram incluídos nesse estudo 4 olhos de 4 pacientes que foram submetidos a trabeculectomia com uso de mitomicina-A e que apresentavam vazamento de humor aquoso com teste de Seidel $+(3$ olhos $)$ ou bolha hiperfiltrante $(1$ olho $)$ resistentes a tratamento clínico e cirúrgico convencional. O tempo póstrabeculectomia não foi considerado como critério de inclusão. Dois pacientes eram do sexo masculino e 2 eram do sexo feminino. A idade média dos pacientes era de 71,25 anos (variação entre 42-92 anos) (Quadro 1).
Os pacientes foram submetidos a uma série de exames iniciais. São eles: avaliação de acuidade visual sem correção, com correção e estenopeico; exame biomicroscópico com fotografias; coloração com fluoresceína; fundoscopia sob midríase (se possível); nos casos de opacidade dos meios, ultrasonografia ocular e de segmento anterior (UBM - Humphrey, EUA); tonometria de aplanação ou medida pelo Tonopen (Oculab, EUA), quando necessário. Todos os pacientes foram devidamente instruídos e assinaram um detalhado consentimento informado sobre o tratamento a que seriam submetidos.

\section{Obtenção e preparação da membrana amniótica}

A obtenção e processamento da membrana amniótica foram realizados de acordo com as normas do protocolo aprovado pela Comissão de Ética da UNIFESP/EPM, baseadas no protocolo proposto por Tseng et al. ${ }^{(8)}$, seguindo as premissas impostas pela United States Food and Drug Administration (FDA) e The American Association of Tissue Banking (AATB).

A membrana amniótica foi obtida a partir das placentas provenientes de cesáreas eletivas de pacientes do Departamento de Obstetrícia e Ginecologia da UNIFESP e Hospital Amparo Maternal, após consentimento assinado pelas parturientes. Todas essas pacientes tinham exames sorológicos negativos para HIV-1, Hepatite B (HBsAg) e sífilis (VDRL).

Após a obtenção da placenta no centro cirúrgico obstétrico, procedia-se sua lavagem com o uso de soro fisiológico $0,9 \%$ em ambiente estéril ${ }^{(8,11)}$. Posteriormente, o âmnio era separado do córion com a utilização de tesoura e pinça estéreis e estendido sobre um filtro de nitrocelulose estéril (Millipore, Bedfort, MA, EUA) com a face epitelial para cima ${ }^{(8,11)}$. A membrana e o filtro foram lavados com solução tampão fosfato contendo $1000 \mathrm{U} / \mathrm{ml}$ de penicilina, $20 \mathrm{mcg} / \mathrm{ml}$ de estreptomicina e $2,5 \mathrm{mcg} / \mathrm{ml}$ de anfotericina $\mathrm{B}$, cortados em fragmentos de aproximadamente $10 \mathrm{X} 10 \mathrm{~cm}$, colocados em um recipiente estéril contendo glicerol (Baxter Healthcare Corporation, Stone

\begin{tabular}{|lcccccc|}
\hline \multicolumn{6}{|c|}{ Quadro 1. Dados dos pacientes com complicações pós-trabeculectomia (TREC) submetidos a transplante de membrana amniótica } \\
N & Sexo & Idade (anos) & Diagnóstico & Tempo entre TREC e cirurgia & Tratamento prévio & Fluoresceína \\
1 & F & 42 & Seidel pós-TREC & 20 dias & 1 sutura, 2 colas & Seidel + \\
2 & M & 69 & Seidel pós-TREC & 2 anos & 4 enxertos, 3 colas, 1 sutura & Seidel + \\
3 & $F$ & 82 & Seidel pós-TREC & 30 dias & 1 sutura \\
4 & M & 92 & Bolha hiperfiltrante cística pós-TREC & 5 anos & Seidel + \\
F=feminino; M=masculino & & & & Seidel - \\
\hline
\end{tabular}

\begin{tabular}{|c|c|c|c|c|c|c|c|c|}
\hline \multicolumn{9}{|c|}{ Quadro 2. Resultados do transplante de membrana amniótica nos pacientes com complicações pós-TREC } \\
\hline $\mathbf{N}$ & AV pré & AV pós & $\begin{array}{l}\text { PIO pré } \\
\text { (mm Hg) }\end{array}$ & $\begin{array}{l}\text { PIO pós } \\
(\mathrm{mm} \mathrm{Hg})\end{array}$ & $\begin{array}{l}\text { Procedimento } \\
\text { associado }\end{array}$ & $\begin{array}{l}\text { Tempo de } \\
\text { seguimento }\end{array}$ & $\begin{array}{l}\text { Tempo de } \\
\text { epitelização }\end{array}$ & Resultados \\
\hline 1 & MM & CD $2 \mathrm{~m}$ & 0 & $14^{*}$ & - & 9 meses & 15 dias & S \\
\hline 2 & MM & MM & 4 & $18^{*}$ & - & 8 meses & 21 dias & $\mathrm{SP}(+$ cola $)$ \\
\hline 3 & MM & MM & 8 & $20^{*}$ & retalho conjuntival & 4 meses & 15 dias & $\mathrm{s}$ \\
\hline 4 & $20 / 200$ & $20 / 200$ & 8 & $18^{*}$ & transplante de esclera & 2 meses & 8 dias & S \\
\hline
\end{tabular}


Mountain, GA, EUA) e meio de preservação de córnea (Ophthalmos, São Paulo) na proporção de $1: 1$ e congelados a $-80^{\circ} \mathrm{C}^{(8,11)}$. Amostras de cada membrana obtida foram enviadas para estudo anátomo-patológico e microbiológico (bacterioscopia e cultura). O tempo máximo para utilização das membranas foi de 3 meses após a congelação ${ }^{(8,11)}$.

\section{Técnica cirúrgica}

Nos casos de vazamento de humor aquoso com Seidel +, foi realizada dissecção da conjuntiva sobre a área da trabeculectomia. Removeu-se todo tecido conjuntival alterado e, em seguida, confeccionou-se fragmento de membrana amniótica do mesmo tamanho da área exposta. Suturou-se o fragmento na episclera com pontos separados de nylon $10.0 \mathrm{e}$, quando necessário (extensa área necrótica e ausência de conjuntiva viável), um segundo fragmento maior que o primeiro foi confeccionado e suturado sobre o primeiro com sutura contínua de nylon 10.0, ancorado na conjuntiva. No caso de lesão extensa e presença de conjuntiva viável, optou-se por colocar um reforço de conjuntiva sobre a(s) membrana(s) amniótica(s).

No caso de bolha hiperfiltrante com afinamento escleral, foi realizada dissecção da conjuntiva na área da bolha cística. Em seguida, após preparo do leito receptor escleral, um fragmento de esclera de cadáver foi confeccionado e suturado na região afinada com Vycril 8.0. Confeccionou-se fragmento de membrana amniótica do mesmo tamanho da área exposta, que foi suturado na episclera com pontos simples de Vycril 8.0 e na conjuntiva com sutura contínua de mononylon 10.0.

Acetato de prednisolona $1 \%$ e ofloxacina tópico foram instilados 3/3horas nas primeiras 2 semanas, decrescendo progressivamente nas próximas semanas.

Estipulou-se que o procedimento seria considerado bemsucedido (S) se houvesse resolução do vazamento da bolha, com aumento da pressão intra-ocular e teste de Seidel negativo, e no caso de bolha hiperfiltrante, com a comprovação biomicroscópica de aumento de espessura da parede e aplanamento da bolha, concomitante com aumento da pressão intraocular do olho afetado. Sucesso parcial (SP) seria considerado no caso de resolução do quadro após introdução de outro procedimento adjuvante (ex: cola). E falência (F) se houvesse recorrência do quadro e necessidade de outro procedimento cirúrgico.

\section{RESULTADOS}

A média do tempo de seguimento foi de 5,75 meses (variação de 2 a 9 meses). Conseguiu-se resolução do quadro em todos os casos (Figuras 1A e 1B). No caso 2, houve persistência do vazamento de humor aquoso com teste de Seidel + após o transplante de membrana amniótica, que necessitou de aplicação de cola e lente de contato para resolução do quadro. Após 3 semanas, a cola desprendeu, tendo sido retirada juntamente com a lente de contato terapêutica. A coloração por fluoresceína desse caso, após a retirada da cola e lente de

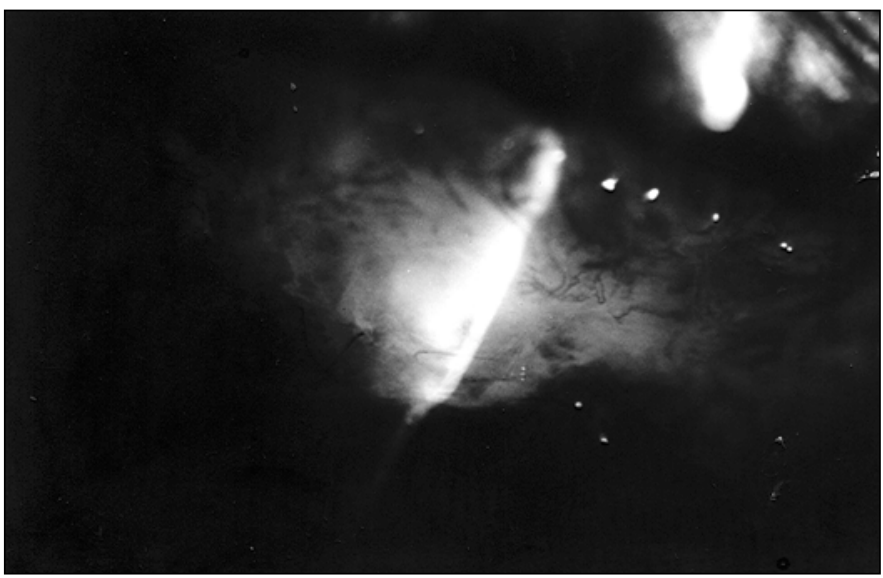

Figura 1 - A) PO 15 dias do caso 1. Nota-se formação de bolha filtrante sob membrana amniótica (seta)

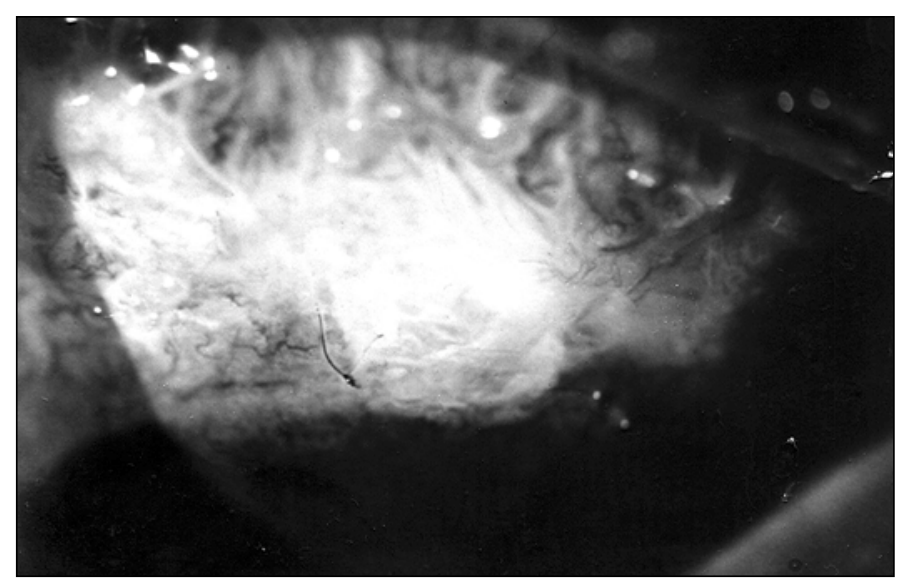

Figura 1 - B) PO 15 dias do caso 1. A coloração por fluoresceína mostra com-pleta epitelização da membrana amniótica e ausência de vazamento

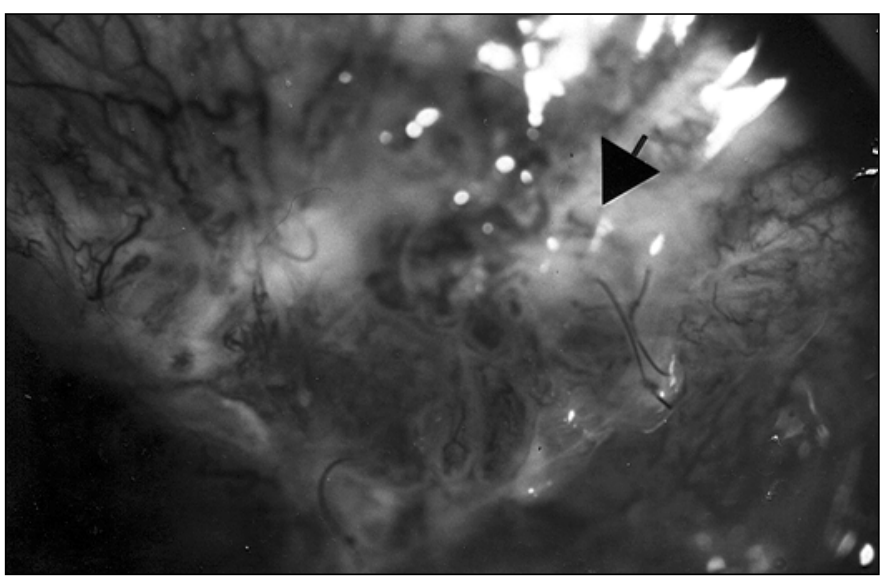

Figura 2 - PO 30 dias do caso 2. Observa-se resolução do Seidel pósTREC e presença de retalho de conjuntiva viável sobre membrana amniótica (seta)

contato, não evidenciou Seidel e demonstrou completa epitelização da bolha filtrante (Figura 2). 
Em média, a epitelização ocorreu em 14,75 dias (variação de 8 a 21 dias). A acuidade visual melhorou em apenas 1 paciente e manteve-se inalterada nos outros 3 casos. Após as cirurgias envolvendo membrana amniótica, a pressão intra-ocular manteve-se sob controle com uso de medicação antiglaucomatosa tópica em todos os pacientes.

\section{DISCUSSÃO}

A membrana amniótica é composta por uma camada de células epiteliais, membrana basal espessa e matriz estroma avascular $^{(8)}$. Sua utilização baseia-se em diversas propriedades, que incluem facilitação no processo de epitelização, redução dos processos inflamatório, angiogênico e cicatricial ${ }^{(8-15)}$. Além disso, possui ação antimicrobiana e é considerada imunologicamente inerte, constituindo uma atraente opção de enxerto ${ }^{(11,16-17)}$.

Neste trabalho, utilizamos membrana amniótica na resolução de complicações pós-trabeculectomia resistentes ao tratamento convencional: 3 casos de bolha com vazamento de humor aquoso e 1 caso de bolha cística hiperfiltrante. Nos casos mais difíceis, utilizamos membrana amniótica associada a retalho de conjuntiva, com objetivo de dar maior suporte e evitar a recidiva. Em um caso, utilizamos com sucesso dois retalhos de membrana amniótica, sem necessidade de manipulação da conjuntiva. Um caso mais grave teve que ter a correção cirúrgica complementada com cola (1 paciente). No caso da bolha cística hiperfiltrante, houve necessidade de enxerto de esclera concomitante, pois havia afinamento escleral importante. Em nenhum caso, entretanto, foi necessário novo procedimento cirúrgico que envolvesse manipulação de conjuntiva ou outro enxerto. Em todos os casos, obteve-se a reconstituição anatômica da superfície da bolha filtrante com manutenção dos valores da pressão intra-ocular com auxílio de medicação antiglaucomatosa. A acuidade visual mantevese inalterada na maioria dos pacientes, que já apresentavam baixa de visão pelo glaucoma.

Muitas das propriedades inerentes à membrana amniótica parecem estar ligadas a presença (e inibição) de fatores de crescimento $^{(11-12,18)}$. Sabe-se que a face estromal da membrana amniótica contém componente(s) que inibem o fator de crescimento beta e sua participação no processo de proliferação e diferenciação dos fibroblastos estromais ${ }^{(19)}$. Mais recentemente, a membrana amniótica tem sido utilizada nas trabeculectomias como substituto dos agentes antimetabólicos para diminuir o processo cicatricial mediado pelos fibroblastos ${ }^{(15)}$.

Como em outras cirurgias com membrana amniótica, observamos um pós-operatório com pouca inflamação ${ }^{(11)}$. A ausência de sintomas no pós-operatório, já relatado por outros autores, chama a atenção ${ }^{(9-14)}$. Em todos os casos houve revascularização do enxerto de membrana amniótica, o que contradiz seu efeito inibidor da neovascularização.

Em resumo, achamos que a membrana amniótica constitui uma opção viável para o tratamento dos casos de bolha com vazamento de humor aquoso e dos casos de bolha cística hiperfiltrante pós-trabeculectomia resistentes ao tratamento convencional. Mais casos são necessários para melhor avaliarmos e refinarmos a técnica operatória.

\section{$S$ UMMAR $\Upsilon$}

Methods: Four eyes of 4 patients presenting post-trabeculectomy with mitomycin-A complications (leaking blebs, 3 eyes, and inadvertent filtering bleb, 1 eye) underwent bleb reconstruction with amniotic membrane. Results: Mean follow-up time was 5.75 months (range, 2 to 9 months). Complete resolution was achieved in all cases. In one case it was necessary to add cyanoacrylate tissue adhesive and place a therapeutic contact lens. The epithelialization occurred within 14.75 days (range, 8 to 21 days). Visual acuity improved in 1 patient and did not change in the other 3 cases. Intraocular pressure remained under control in all patients with the additional use of topical antiglaucomatous medication. Conclusions: The use of amniotic membrane seems to represent a viable option for the treatment of post-trabeculectomy leaking and inadvertent filtering blebs that were resistant to conventional therapy. More cases are necessary for further analysis and to refine the technique.

Keywords: Biological dressings; Trabeculotomy/adverse effects

\section{REFERENCIAS}

1. Geyer O. Management of large, leaking, and inadvertent filtering blebs with the neodymium: YAG Laser. Ophthalmology 1998;105:983-7.

2. Zalta AH, Wieder RH. Closure of leaking filtering blebs with cyanoacrylate tissue adhesive. Br J Ophthalmol 1991;75:170-3.

3. Cleasby GW, Fung WE, Webster RG. Cryosurgical closure of filtering blebs. Arch Ophthalmol 1972;87:319-23.

4. Wilson MR, Kotas-Neumann R. Free conjunctival patch for repair of persistent late bleb leak. Am J Ophthalmol 1994;117:569-74.

5. Scheie HG, Guehl JJ. Surgical management of overhanging blebs after filtering procedures. Arch Ophthalmol 1979;97:325-6.

6. Smith MF, Magauran R, Doyle JW. Treatment of postfiltration bleb leak by bleb injection of autologous blood. Ophthalmic Surg 1994;25:636-7.

7. O'Connor DJ, Tressler CS, Caprioli J. A surgical method to repair leaking filtering blebs. Ophthalmic Surg 1992;23:336-8.

8. Kim JC, Tseng SC. Transplantation of preserved human amniotic membrane for surface reconstruction in severely damaged rabbit corneas. Cornea 1995; 14:473-84.

9. Tsubota K, Satake Y, Ohyama M, Toda I, Takano Y, Ono M, Shinozaki N, Shimazaki J. Surgical reconstruction of the ocular surface in advanced ocular cicatricial pemphigoid and Stevens-Johnson syndrome. Am J Ophthalmol 1996;122:38-52.

10. Shimazaki J, Yang HY, Tsubota K. Amniotic membrane transplantation for ocular surface reconstruction in patients with chemical and thermal burns. Ophthalmology 1997;104:2068-76.

11. Gomes JAP, Komagome CM, Santos N, Chaves AP, Cunha MC, Freitas D. Membrana amniótica nas cirurgias de reconstrução da superfície ocular nas Ceratoconjuntivites Cicatriciais - Resultados preliminares. Arq Bras Oftalmol 1999;62:562-76.

12. Lee S, Tseng SC. Amniotic membrane transplantation for persistent epithelial defects with ulceration. Am J Ophthalmol 1997;123:303-12. 
13. Prabhasawat P, Barton K, Burkett G, Tseng SC. Comparison of conjunctival autografts, amniotic membrane grafts and primary closure for pterygium excision. Ophthalmology 1997;104:974-85.

14. Tseng SC, Prabhasawat P, Lee S-L. Amniotic membrane transplantation for conjunctival surface reconstruction. Am J Ophthalmol 1997;124:765-74.

15. Barton K, Budenz DL, Khaw PT, Tseng SC. Amniotic membrane transplantation in glaucoma surgery [abstract]. Invest Ophthalmol Vis Sci 1997;38:S473.

16. Talmi YP, Sigler L, Inge E. Antibacterial properties of human amniotic membranes. Placenta 1991;12:285-6.
17. Adinolfi M, Akle CA, McColl I. Expression of HLA antigens, $\beta_{2}$-microglobulin amd enzymes by human amniotic epithelial cells. Nature 1982;295: $325-7$

18. Gomes JAP, Komagome CM, Pena JDO, Santos N, Chaves AP, Freitas D. Clinical and ultrastructural aspects of amniotic membrane for ocular surface reconstruction [ abstract]. Invest Ophthalmol Vis Sci 1999;40:S329.

19. Tseng SG, Lee SB, Li DQ, Tan DT. Supression of TGF-beta signaling in both normal conjunctival fibroblasts and pterygial body fibroblasts by amniotic membrane [abstract]. Invest Ophthalmol Vis Sci 1999;40:S579. 